\title{
Imageamento GPR 3D de alvos forenses na área do sítio controlado de criminalística (SITCRIM)
}

\author{
Daniel Sobreira de Oliveira Buso* (danielbuso.geo@gmail.com - Graduando de Geofísica do IG/UnB) \\ Marcelo de Lawrence Bassay Blum (blum.mlbb@dpf.gov.br - Perito Criminal Federal INC/DPF) \\ Welitom Rodrigues Borges (welitom@unb.br - Docente do IG/UnB)
}

Copyright 2016, SBGf - Sociedade Brasileira de Geofísica

Este texto foi preparado para a apresentação no VII Simpósio Brasileiro de Geofísica, Ouro Preto, 25 a 27 de outubro de 2016. Seu conteúdo foi revisado pelo Comitê Técnico do VII SimBGf, mas não necessariamente representa a opinião da SBGf ou de seus associados. É proibida a reprodução total ou parcial deste material para propósitos comerciais sem prévia autorização da SBGf

\section{Abstract}

This paper presents results of a geophysical survey carried out in the Controlled Criminalistics Test Site (SITCRIM), created in 2012 by the National Institute of Forensic Science, from the Department of Federal Police (INC / DITEC / DPF), located in Brasília-DF. The aim of this study was to provide reflection patterns of some buried forensic targets such as metallic barrels, plastic drums by using GPR.

\section{Introdução}

Um dos campos da investigação forense prende-se com a localização de provas ocultadas no subsolo onde as buscas através de escavações são inviáveis (estruturas complexas, risco de destruição de evidências, dimensão das áreas de investigação, etc.). A investigação em geociências forense tem por missão localizar, identificar, recolher e catalogar essas provas (físicas) com a finalidade de apresentá-las em tribunal (LOURENÇO, 2009).

A geofísica, dentro das geociências possui um importante papel na pesquisa forense, uma vez que investiga o meio sem modificá-lo. Dentre os métodos geofísicos usados em perícias forenses, destaca-se o radar de penetração no solo (Ground Penetrating Radar - GPR).

Com o objetivo de minimizar as ambiguidades de investigações forenses e de possibilitar o treinamento contínuo de peritos brasileiros, o Instituto Nacional de Criminalística do Departamento de Polícia Federal (INC/DITEC/DPF), implantou em 2012, o Sítio Controlado de Criminalística (SITICRIM, Figura 1; BLUM e RUSSO, 2012).

O SITCRIM compreende uma área de $625 \mathrm{~m}^{2}$ e dispõe de seis trincheiras contendo objetos diversos cujo intuito é de simular situações corriqueiras das áreas de aplicação da geofísica forense, como ossadas bovinas, cerâmicas e artefatos arqueológicos, projéteis e armamentos, entre outros.

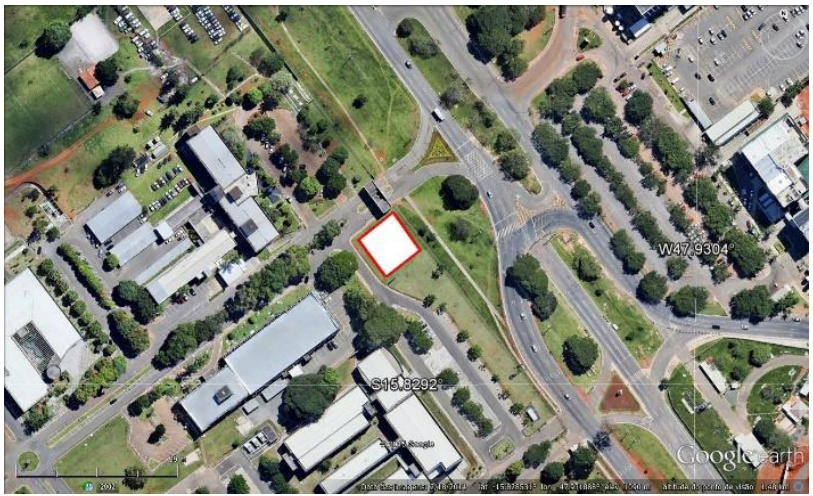

Figura 1 - Imagem com a localização do SITCRIM, Brasília/DF (quadrado branco).

\section{Metodologia}

O GPR é um método geofísico eletromagnético que se baseia na propagação de ondas de rádio em altas frequências (10 MHz a $3 \mathrm{GHz}$ ) para identificar estruturas rasas (DAVIS e ANNAN, 1989; PORSANI, 1999). O sistema consiste em uma unidade de controle e um conjunto de antenas. Uma antena transmissora que irradia pulsos eletromagnéticos em direção ao subsolo, e uma antena receptora que capta o sinal refletido. Os sinais refletidos são registrados por sistemas digitais em função do tempo de percurso medido em nanossegundos (ns), entre a onda emitida e a recebida (DAVIS e ANNAN, 1989).

A propagação das ondas eletromagnéticas depende da frequência do sinal transmitido e das propriedades elétricas (condutividade elétrica, permissividade dielétrica e permeabilidade magnética) dos materiais presentes no meio.

O objetivo desta pesquisa foi verificar os padrões de reflexão de barris metálicos e tambores plásticos, com resultados 3D de GPR, dispostos em diferentes posições e profundidades em uma das trincheiras cuja dimensão é 1,5 metros de largura por 10 metros de comprimento e 1,5 metros de profundidade (figuras 2 e 3 ). 


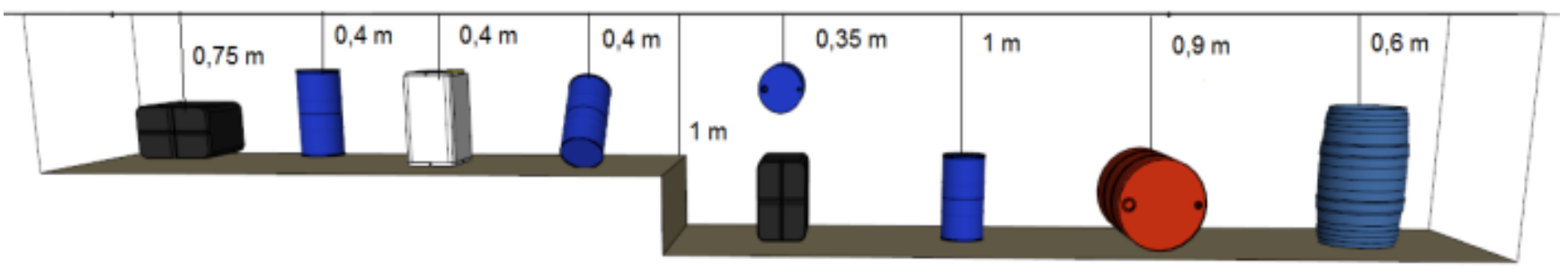

(a)

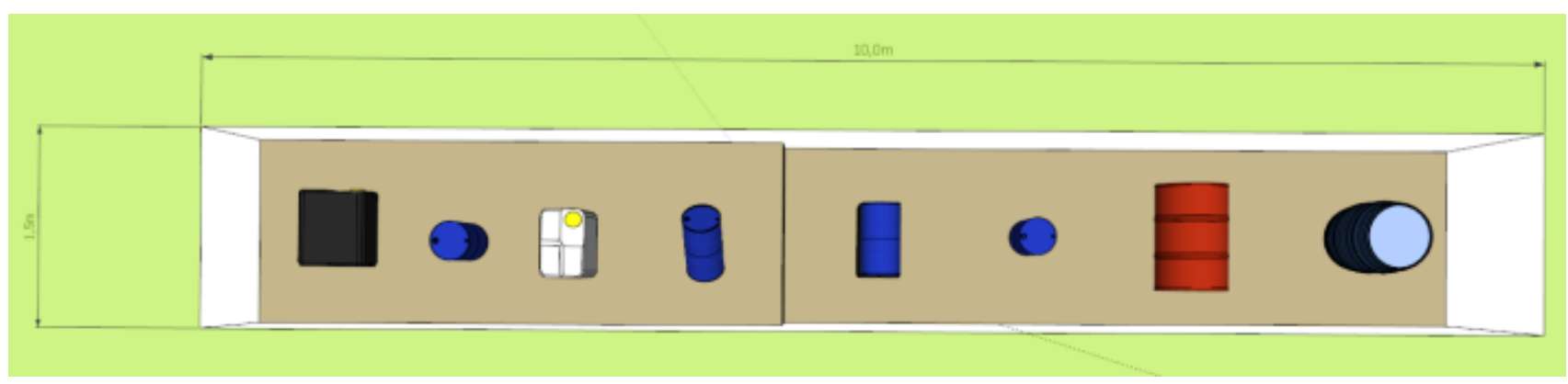

(b)

Figura 2 - Croqui da trincheira com os tambores metálicos e plásticos dispostos na área do SITCRIM. (a) vista lateral. (b) vista em planta.

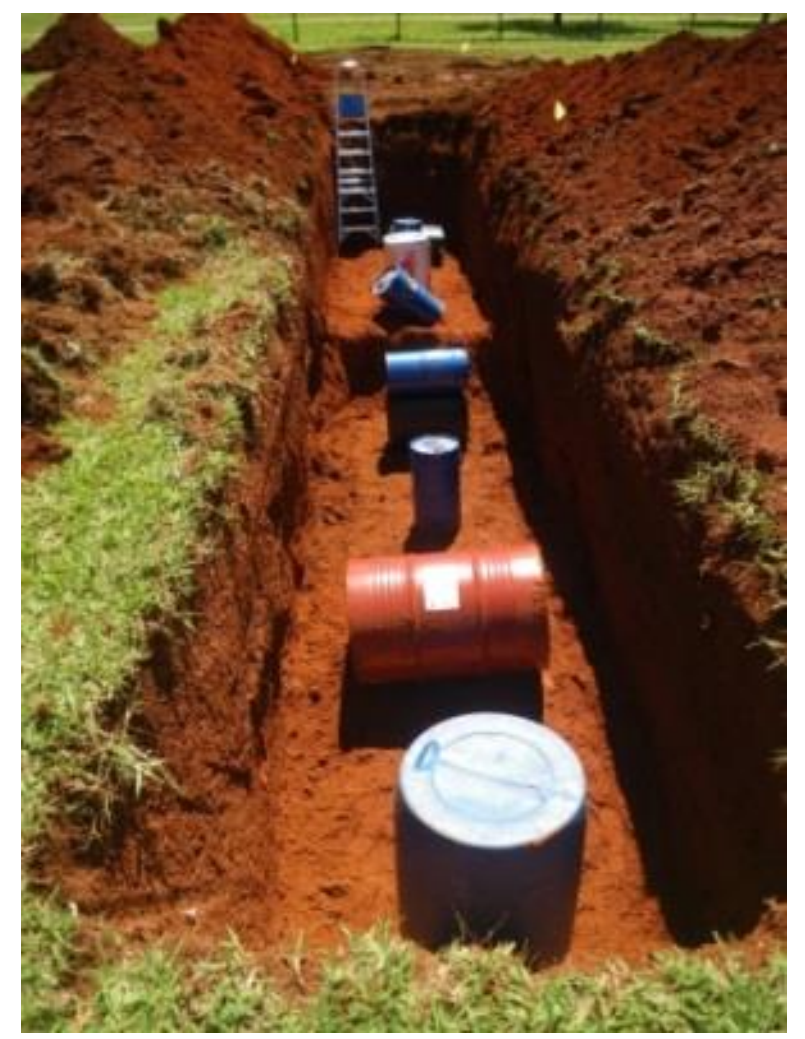

Figura 3 - Foto evidencia os tambores metálicos e plásticos enterrados na área do SITCRIM.

\section{Aquisição}

$\mathrm{Na}$ aquisição de dados de GPR usou-se o sistema SIR3000 acoplado a antenas blindadas de $400 \mathrm{MHz}$. Usou-se a frequência de $400 \mathrm{MHz}$ em função da optimização de boa profundidade e boa resolução, e por ser a frequência mais utilizada na perícia forense.

Optou-se por realizar as aquisições paralelas ao eixo principal da trincheira, em função da disposição dos alvos ao longo da mesma. Em campo, realizaram-se perfis equidistantes em $10 \mathrm{~cm}$, ao longo de uma área de $34 \mathrm{~m}^{2}$ $(3,4 \mathrm{~m} \times 10 \mathrm{~m})$, o que totalizou em 34 perfis de GPR 2D (Figura 4).

Os parâmetros de aquisição de dados de GPR foram: janela temporal de $80 \mathrm{~ns}$, frequência de amostragem de $6375 \mathrm{MHz}$, amostragem espacial de $2 \mathrm{~cm}$, amostragem temporal de 0,078ns, e 1024 amostras por traço.

No processamento de dados usou-se o software REFLEXW, versão 7.5 (Sandmeier, 2015). No processamento de dados 2D utilizou-se o Módulo 2Ddataanalysis e as respectivas etapas: ajuste do tempo zero (identificação da primeira chegada da onda direta no solo, para situar os refletores com relação ao tempo no solo), aplicação de ganhos para recuperação da perda de energia provocada pelo espalhamento geométrico da onda eletromagnética no solo (energy decay), filtragem 1D dos ruídos aleatórios de baixa e alta frequência (bandpass trapezoidal), filtragem 2D para remoção dos ruídos coerentes (em função das características do alvo em questão, a remoção de refletores contínuos e 
horizontais auxilia na determinação dos alvos pontuais difrações), migração dos refletores hiperbólicos para disposição dos refletores em sua posição correta.

Com o conhecimento das profundidades dos alvos, e o tempo de ocorrência das difrações relacionadas aos mesmos, calculou-se a velocidade de propagação da onda eletromagnética a partir da Equação 1.

$$
v=\frac{2 p}{\Delta t}
$$

(Equação 1)

Em que $p$ é a profundidade e $\Delta t$ é o tempo em $n s$ de propagação do sinal. As velocidades obtidas para o meio variaram entre 0,07 a $0,09 \mathrm{~m} / \mathrm{ns}$, o que confirma a variabilidade do solo em função do atual grau de compactação.

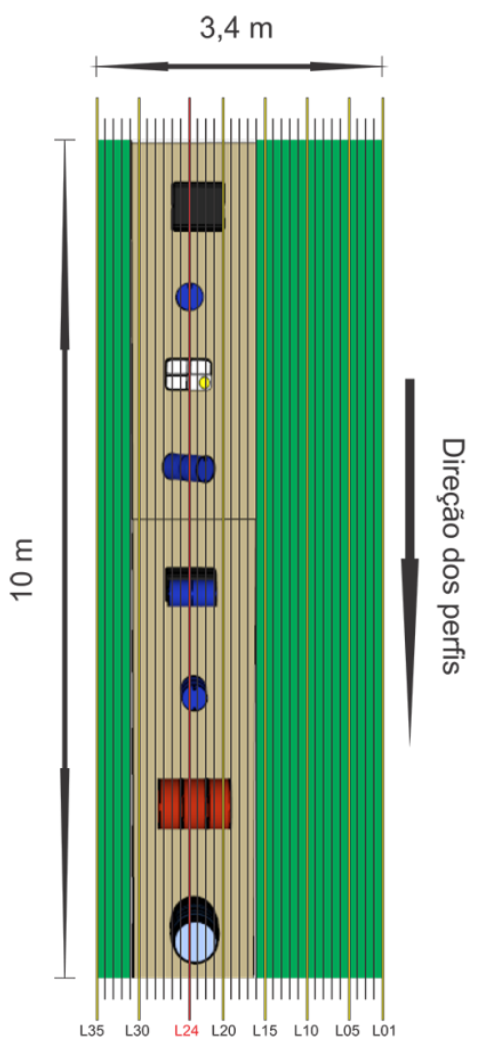

Figura 4 - Croqui com a disposição dos perfis e a posição da trincheira e respectivos alvos.

\section{Resultados}

Inicialmente para verificar os resultados de GPR optou-se pela análise do processamento realizado em 2D. Deste modo usou-se a L24 para esta análise em função da mesma cruzar o centro dos alvos dispostos na trincheira. $\mathrm{Na}$ Figura $\mathbf{5 a}$ notam-se as hipérboles relacionadas a cada alvo, as maiores hipérboles representam os maiores alvos. Na migração todas as hipérboles colapsaram, o que evidenciou com maior clareza o posicionamento correto dos alvos (Figura 5b).
No bloco de GPR 3D realizaram-se vários cortes para verificar o posicionamento dos alvos, bem como se os mesmos encontram-se na profundidade estimada.

Em função da composição mineralógica do solo (argiloso), todos os alvos mostram alta impedância elétrica, principalmente porque se encontram vazios.

Na Figura 6, corte realizado na profundidade de 0,6 metros, nota-se a anomalia relacionada ao tambor posicionado de modo vertical. $\mathrm{Na}$ Figura 7, corte realizado na profundidade de 0,9 metros, registram-se as anomalias relacionadas a dois tambores metálicos. No corte realizado na profundidade de 1,1 metros notam-se as anomalias relacionadas a um tambor quadrado, e outros dois tambores verticalizados (Figura 8).

Na Figura 9, corte realizado na profundidade de 1,4 metros, notam-se dois tambores plásticos, um de formato quadrado e outro horizontalizado, localizado abaixo do metálico como exposto em croqui.

\section{Conclusão}

Os resultados de GPR obtidos neste trabalho confirmam a aplicabilidade do método na identificação de alvos forenses (tambores plásticos e metálicos com possíveis resíduos), visto que todos os alvos foram identificados nos registros realizados com a antena blindada de $400 \mathrm{MHz}$. Todavia, nota-se que a forma dos alvos não foi bem mapeada, principalmente pelo erro causado pelo mau posicionamento dos perfis de GPR 2D, o que evidencia ainda mais a necessidade de uma aquisição acurada, pois em situações reais, erros de posicionamento de alvos podem ser cruciais para a danificação ou perda dos mesmos.

\section{Agradecimentos}

Ao Departamento de Polícia Federal do Instituto Nacional de Criminalística por autorizar o desenvolvimento da pesquisa na área do SITCRIM.

\section{Referências}

BLUM, MLB. e RUSSO, D. 2012. SITICRIM - Brazilian Test Site for Forensic Geophysical Research. In: European Academy of. Forensic Science Conference, The Hague: EAFS 2012.

DAVIS, J.L; ANNAN, A.P., 1989. Ground penetrating radar for high resolution mapping of soil and rock stratigraphy. Geophysical prospecting, Vol. 37: 531-551.

LOURENÇO, VL. 2009. Utilização do Radar de Penetração nos Solos (GPR) na detecção de estruturas no âmbito nas Ciências Forenses. Trabalho de Conclusão de Curso (Centro de Geofísica), Universidade de Évora, Portugal, 44pp.

PORSANI, J. L., 1999. Ground Penetrating Radar (GPR): Proposta metodológica de emprego em estudos geológico-geotécnicos nas regiões de Rio Claro e Descativado - SP, Tese de Doutorado, Instituto de Geociências e Ciências Exatas, UNESP, Campus de Rio Claro - SP, 145p. 

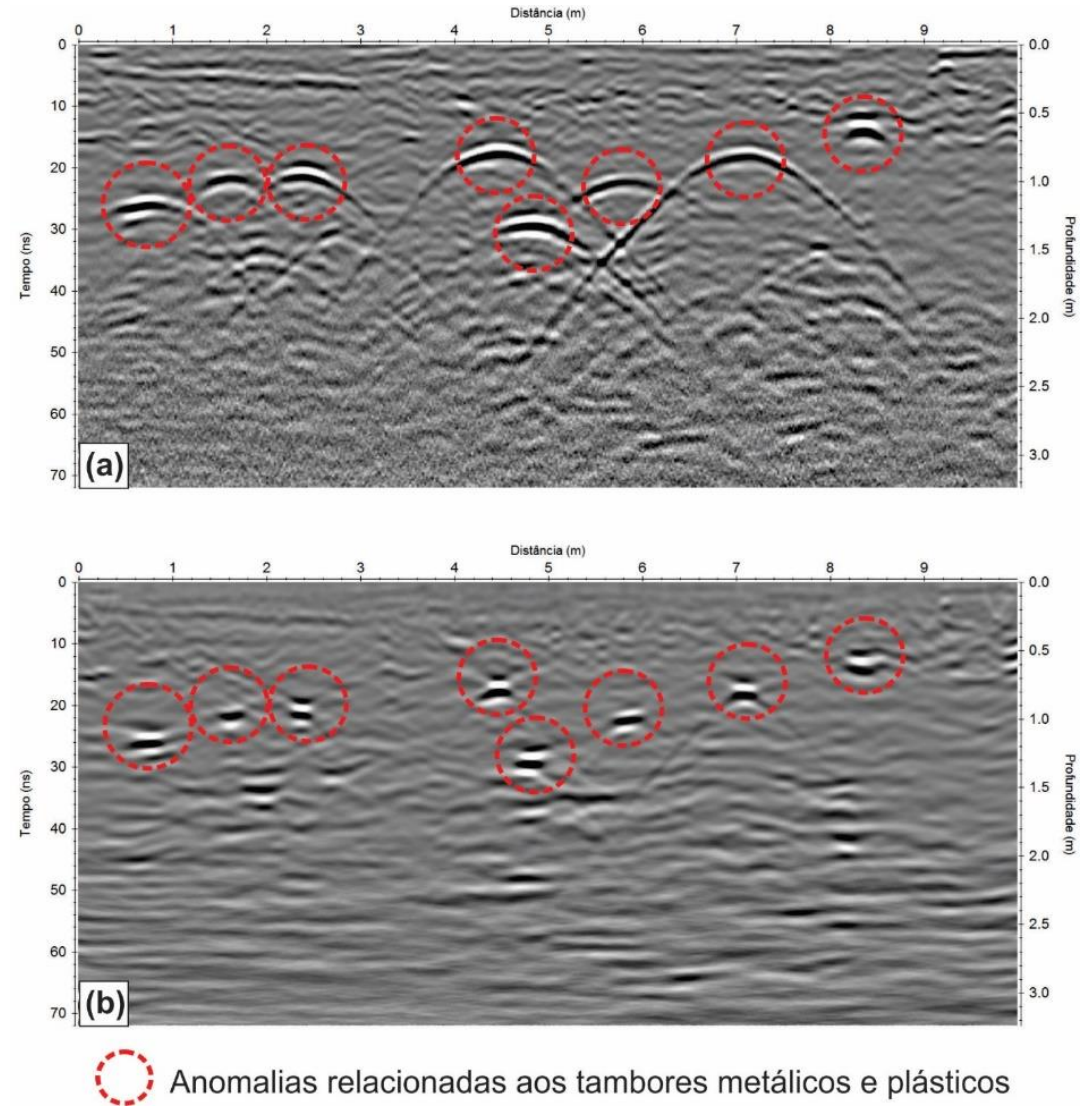

Figura 5 - Seção de GPR (L24) que cruza o centro dos tambores metálicos e plásticos enterrados na trincheira do SITCRIM. (a) Seção não migrada. (b) Seção migrada.
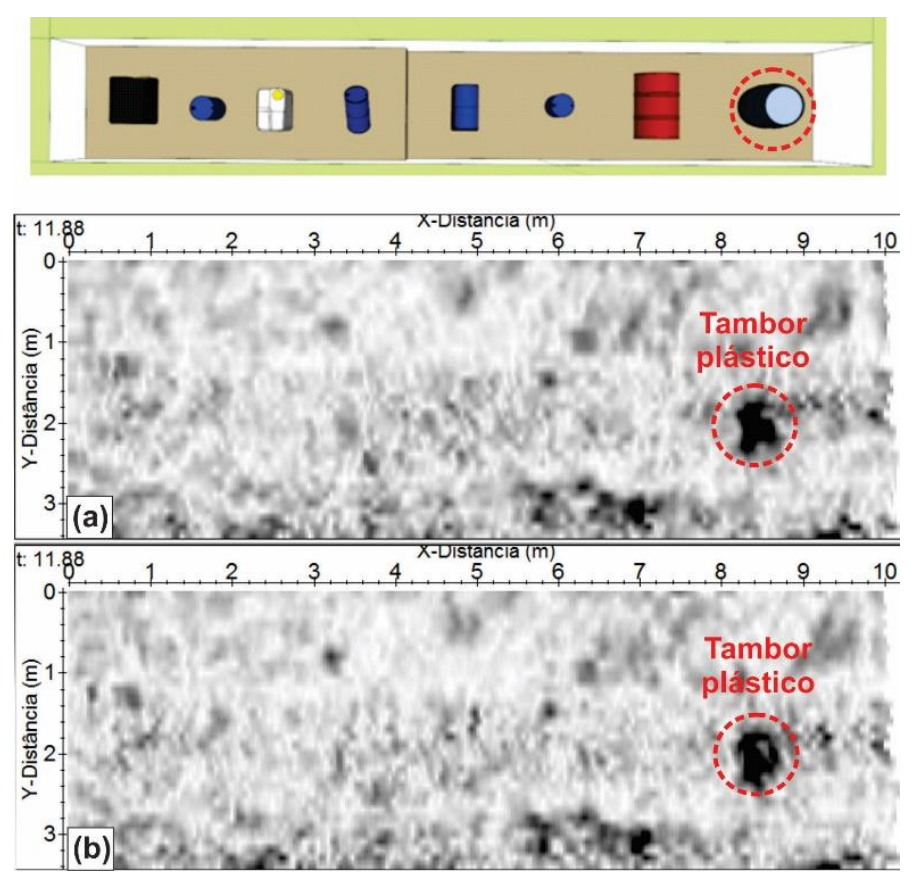

Figura 6 - Cortes na profundidade de 0,6 metros, realizados no bloco de GPR 3D, com a anomalia relacionada ao tambor plástico. (a) Dado não migrado. (b) Dado migrado. 

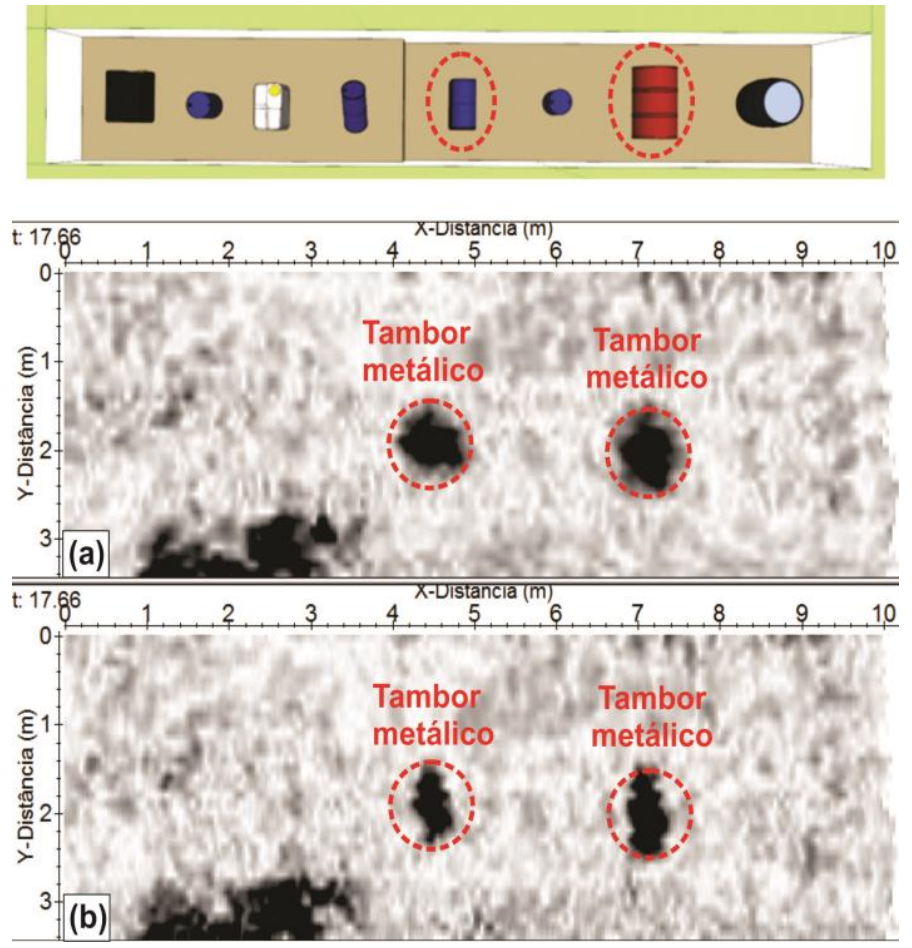

Figura 7 - Cortes na profundidade de 0,9 metros, realizados no bloco de GPR 3D, com a anomalia relacionada ao tambor plástico. (a) Dado não migrado. (b) Dado migrado.

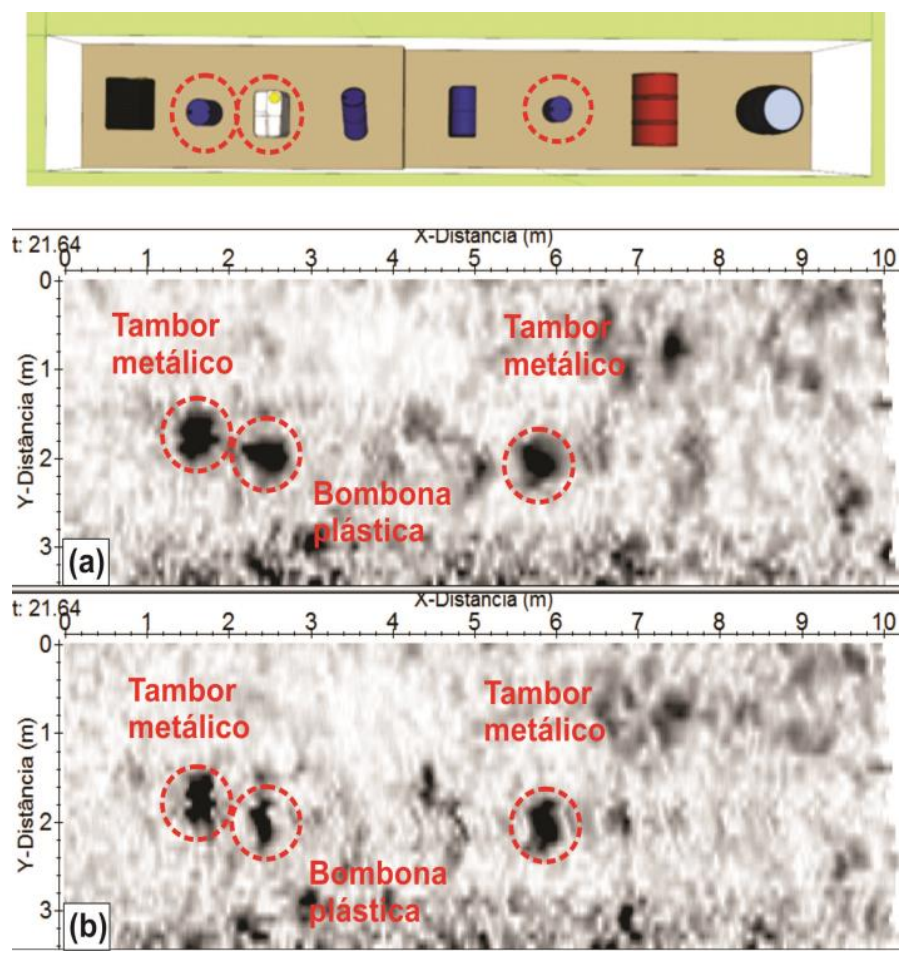

Figura 8 - Cortes na profundidade de 1,1 metros, realizados no bloco de GPR 3D, com a anomalia relacionada ao tambor metálico, bombona plástica e tambor metálico, da esquerda para direita, respectivamente. (a) Dado não migrado. (b) Dado migrado. 

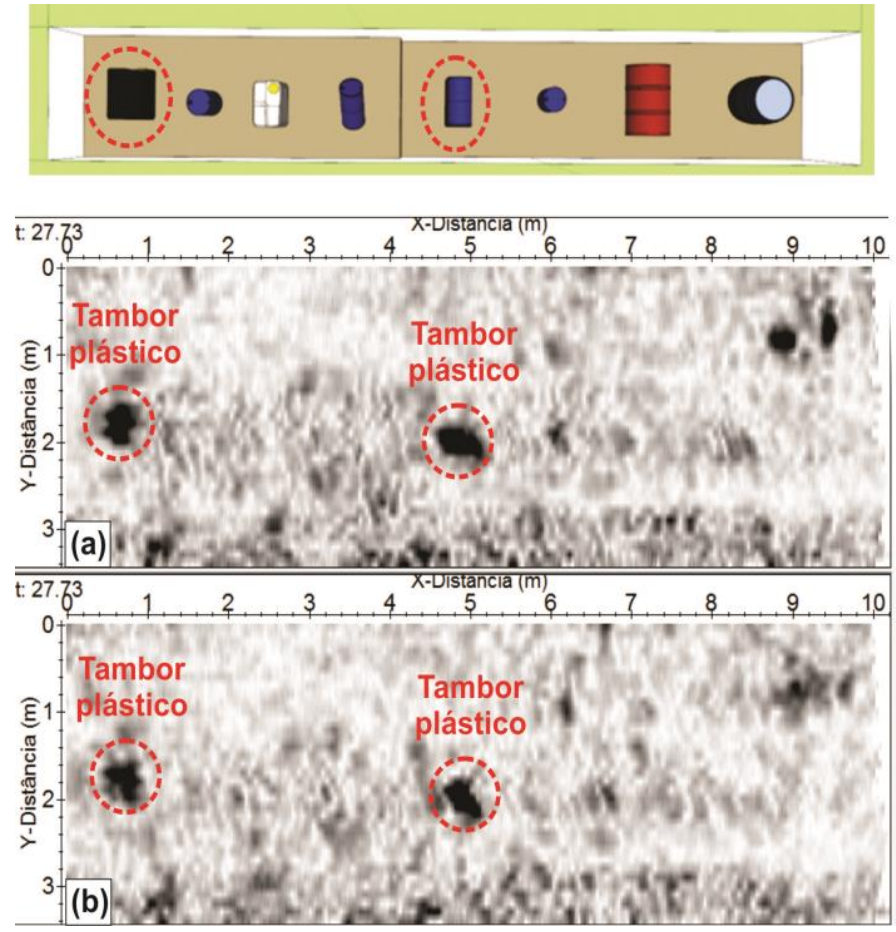

Figura 9 - Cortes na profundidade de 1,4 metros, realizados no bloco de GPR 3D, com a anomalia relacionada aos tambores plásticos. (a) Dado não migrado. (b) Dado migrado. 